\title{
Treatment of Homozygous Familial Hypercholesterolemia: Challenges and Latest Development
}

\author{
Min-Ji Charng \\ Additional information is available at the end of the chapter
}

http://dx.doi.org/10.5772/63949

\begin{abstract}
Familial hypercholesterolemia (FH) is an autosomal codominant genetic disorder of lipoprotein metabolism. Patients can be heterozygous $(\mathrm{HeFH})$ with one mutated allele, homozygous $(\mathrm{HoFH})$ with two identical mutations, or compound heterozygous with different mutations in each allele. HoFH is the more severe form of the disease and is associated with extremely elevated levels of total cholesterol and low-density lipoprotein cholesterol (LDL-C). These lipid abnormalities are associated with accelerated atherosclerosis and cardiovascular disease (CVD) and an increased risk of cardiac events and early death. The prevalence of HoFH has been estimated to be 1 in 1 million; however, this is likely an underestimation as the disease is substantially underdiagnosed and undertreated. Early diagnosis and treatment are important to reduce CVD events. Aggressive therapy with conventional agents such as statins and ezetimibe produce substantial reductions in LDL-C, but patients rarely reach target goals. Apheresis should be considered in all patients with $\mathrm{HoFH}$, although LDL-C levels rapidly rebound to baseline levels. Three recently introduced novel agents (mipomersen, lomitapide, and evolocumab) - each with a unique mechanism of action-have increased therapeutic options in this difficult-to-treat population. When added to standard therapy, these agents produce significant additional LDL-C lowering and can potentially improve clinical outcomes.
\end{abstract}

Keywords: evolocumab, familial hypercholesterolemia, lomitapide, mipomersen, treatment 


\section{Introduction}

Familial hypercholesterolemia $(\mathrm{FH})$ is an autosomal codominant genetic disorder of lipoprotein metabolism, usually caused by mutations in the low-density lipoprotein (LDL) receptor ( $L D L R)$ gene or other genes that affect LDLR function. Patients can be heterozygous (HeFH) with one mutated allele, homozygous (HoFH) with two identical mutations, or compound heterozygous with different mutations in each allele [1]. Patients with HoFH have either a complete absence or marked impairment (i.e., 2-30\% activity) in LDLR function [1]. There are a number of defects in lipid metabolism among patients with FH that include reduced LDLR-mediated catabolism of LDL, impairment of apolipoprotein B (apo B)-mediated clearance of LDL, and increased proprotein convertase subtilisin/kexin type 9 (PCSK9) levels, which mediates posttranslational destruction of LDLRs [2,3].

Since the reduction of LDLRs in HoFH is more pronounced than that seen with $\mathrm{HeFH}$, hypercholesterolemia is usually more severe in $\mathrm{HoFH}$ than in $\mathrm{HeFH}$ and is characterized by very high serum levels of total cholesterol and LDL-cholesterol (LDL-C). Levels of LDL-C are typically above $500 \mathrm{mg} / \mathrm{dL}$ and total cholesterol levels range from 650 to $1000 \mathrm{mg} / \mathrm{dL}$ when $\mathrm{HoFH}$ is untreated, whereas LDL-C levels are typically greater than $300 \mathrm{mg} / \mathrm{dL}$ when treated [2-5]. High-density lipoprotein cholesterol (HDL-C) is often decreased and triglyceride levels are generally normal [4].

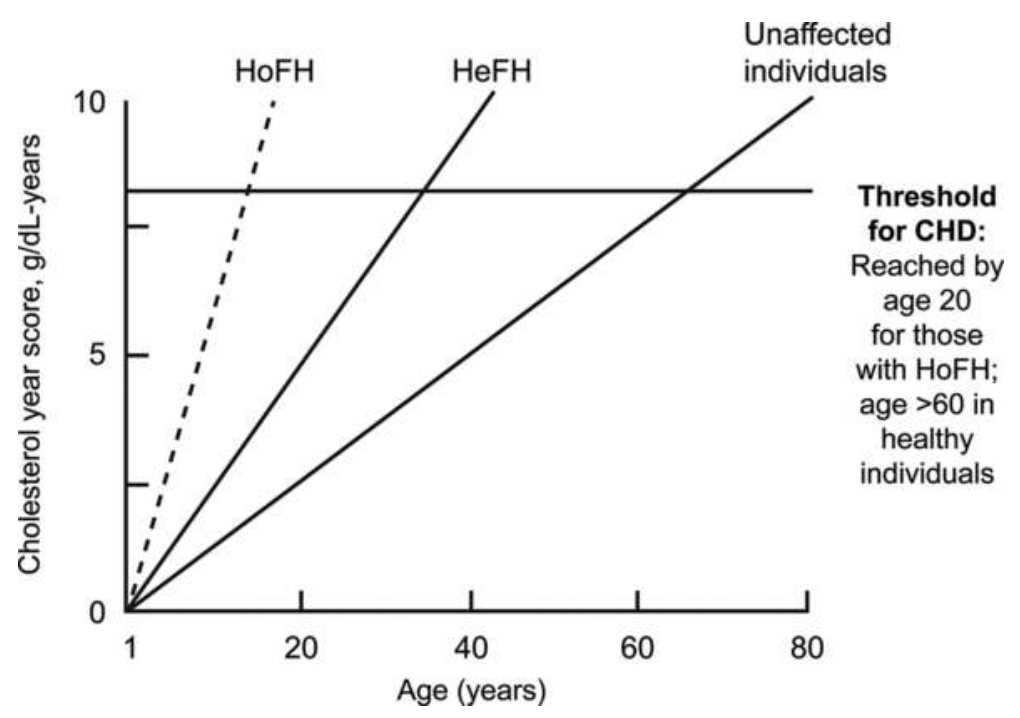

Figure 1. Cumulative LDL exposure in patients with FH [8, 9]. Modified from Horton et al. 2009 [9].

The severe lipid abnormalities associated with $\mathrm{HoFH}$ result in accelerated atherosclerosis, accelerated cardiovascular disease (CVD), and an increased risk of cardiac events and early death. It is estimated that CVD risk is increased by up to 20-fold in untreated patients and still 
elevated approximately 10-fold in patients receiving statins [5-7]. The lifelong exposure of highly elevated lipid levels means that signs/symptoms of CVD occur at an early agetypically prior to 20 years of age and as early as preteen years with the highest risk in males $[5,8]$. Females develop CVD about 10 years later than males [6]. Young patients often have severe and widespread atherosclerosis in all major arterial beds, including the carotid, coronary, femoral, and iliac, and there have been instances of acute myocardial infarction and sudden death in patients as young as 4 years of age [8]. The CVD risk is related to cumulative LDL-C exposure. As seen in Figure 1, patients with HoFH exceed the theoretical threshold of LDL-C exposure in early childhood compared with early middle age for patients with $\mathrm{HeFH}$ and after age 60 years for normal healthy individuals [8,9]. Although, as with all individuals, the risk of developing CVD is also related to the presence of other genetic or environmental risk factors, the effect of each risk factor is amplified in the setting of dramatically elevated cholesterol levels [4].

The physical signs and symptoms of HoFH are characterized by accelerated atherosclerosis and the deposition of cholesterol. Atherosclerotic manifestations include vascular endothelial damage that produces premature coronary artery disease (CAD), peripheral artery disease, and valvular disease (e.g., aortic stenosis) [4]. Deposition of cholesterol results in the development of cutaneous or tendinous xanthomas and corneal arcus [8]. Xanthomas typically occur around the eyelids and tendons of the feet, hands, and elbows [5].

$\mathrm{HoFH}$ is substantially underdiagnosed and undertreated [7]. For example, it is estimated that less than $1 \%$ of patients with $\mathrm{FH}$ are diagnosed in most countries and that only $48 \%$ of patients with FH were receiving statin therapy in one Danish study [7]. Most patients with FH are not identified because of inconsistent screening and general unawareness [6]. Indeed, the disease is often not recognized until the initial cardiovascular (CV) event [6].

\section{Epidemiology}

The exact prevalence rate of $\mathrm{HoFH}$ is unknown. Although the prevalence is historically estimated to be approximately 1 in 1 million [7], this likely underestimates true prevalence rates. More recent estimates, based on surveys of unselected general populations that found a prevalence of HeFH of 1 in 200 or 1 in 244, suggest a prevalence of 1 in 160,000 to 1 in 300,000 for HoFH [10]. Founder mutations that reduce genetic variation can influence the prevalence in certain racial groups or geographic locations, resulting in increased prevalence in certain groups (e.g., French Canadian, the Netherlands, Lebanese, Hokuriku district of Japan, South African Afrikaners) [11-15]. National programs that include patient registries and cascade screening have been useful for identifying patients and facilitating treatments.

\section{Genetics}

True HoFH is caused by two identical mutations that are inherited in an autosomal dominant pattern [16]. Two mutant alleles of the LDLR gene (MIM 606945) cause the majority (85-95\%) 
of cases $[7,10,17]$. Mutations in this gene cause a reduction in LDLR activity and are associated with decreased clearance of LDL particles and increased LDL-C levels.

Secondary genes associated with HoFH include APOB (MIM107730), PCSK9 (MIM 607786), and LDLR-adaptor protein 1 (LDLRAP1; MIM 605747) [8, 10, 17]. In addition to "true" HoFH, patients with $\mathrm{HoFH}$ can have compound heterozygous mutations (different mutations in each allele of the same gene) or double heterozygous mutations (mutations in two different genes affecting LDLR function) [7, 10]. The severity of the HoFH depends on residual LDLR activity. Irrespective of the underlying genetic defect, patients with $\mathrm{HoFH}$ are classified as either receptor negative (i.e., $<2 \%$ residual activity) or receptor defective (i.e., $2-25 \%$ residual activity) [10]. The effect on LDL-C concentrations is also related to genotype. Homozygous $L D L R$-defective mutations are generally associated with the highest LDL-C levels, followed by compound heterozygous $L D L R$-defective $+L D L R$-negative mutations, homozygous LDLRAP1 or LDLR-defective mutations, homozygous APOB or PCKS9 gain-of-function mutation, and double heterozygous mutation $[5,10]$. Metabolic defects include impaired LDL uptake (the most common functional defect), hepatic oversecretion of apo B, decreased catabolism of triglyceride-rich lipoproteins, increased plasma levels of lipoprotein(a) (Lp(a)), and low levels of HDL-C [10].

\section{Diagnosis}

Since CV risk is related to the cumulative exposure to elevated lipids, early diagnosis is important for earlier treatment of $\mathrm{HoFH}$ to reduce $\mathrm{CV}$ risk. Although genetic testing can confirm $\mathrm{FH}$, it is not well defined since genetic confirmation can be difficult to verify in some patients [10]. Indeed, genetic testing is generally not needed as the disease is primarily diagnosed via clinical and biochemical features $[6-8,10,18]$. A number of diagnostic criteria have been proposed [8], but they are typically based on family history (i.e., $\mathrm{HeFH}$ in both parents and/or premature $\mathrm{CAD}$ ), the presence of physical manifestations (i.e., tendon xanthomas, corneal arcus) at an early age, severely increased LDL-C, and molecular diagnosis. Patients with HoFH generally have untreated LDL-C levels $>500 \mathrm{mg} / \mathrm{dL}(>13 \mathrm{mmol} / \mathrm{L})$ or treated levels $\geq 300 \mathrm{mg} / \mathrm{dL}$ ( $\geq 7.76 \mathrm{mmol} / \mathrm{L}$ ) [8]. However, not all patients (especially children) with HoFH have significantly elevated LDL-C, with more than one-half of Dutch children with HoFH having LDL-C levels between 217 and 379 mg/dL (5.6-9.8 mmol/L) [10]. Patients with a suspected diagnosis of $\mathrm{HoFH}$ should typically be referred to a specialized center for proper comprehensive management $[6,10]$.

Since early detection of patients with HoFH is crucial for the prevention of CVD, targeted and cascade (i.e., identifying family members at risk) screening is recommended for the identification of new cases in adults $[6,7,16,19,20]$. Targeted screening to identify index cases is recommended for patients with hypercholesterolemia and at least one of the following features: personal/family history of xanthomas or premature CVD or family history of significant hypercholesterolemia or sudden premature cardiac death [6, 7]. Specific criteria in Europe (i.e., European Atherosclerosis Society [EAS]) are similar, but somewhat different than 
those of the National Lipid Association in the United States (US), with slightly different cholesterol cut-points for screening [21]. Such testing is important because most patients identified via screening were not aware of the diagnosis and were therefore not receiving therapy [17]. The index subject should be referred for genetic screening and a family pedigree should be created to identify potential cases, followed by cascade screening with LDL-C measurements [7]. Targeted screening is also recommended in children and adolescents with $\mathrm{CV}$ risk factors $[6,16]$. Prenatal diagnosis is possible, and it is recommended that the partners of known cases of HeFH should be tested to exclude the disease [22]. Economic modeling has shown that comprehensive screening using cholesterol and DNA testing is cost-effective [19].

\section{Treatment options}

Given the severity of hypercholesterolemia with increased CV risk, HoFH requires intensive therapy. However, HoFH is often unresponsive to traditional treatment [20]. A number of societies and associations in the United States (American College of Cardiology/American Heart Association; National Lipid Association) [20, 23, 24], Europe (EAS; National Institute for Health and Care Excellence) [10, 25], and Canada (Canadian Cardiovascular Society) [6] have published guidelines on the treatment of HoFH. The primary target of treatment in these guidelines is the reduction of LDL-C via a combination of lifestyle, antihyperlipidemic pharmacotherapy, and apheresis [6, 10, 20, 23, 26]. Since lipid-lowering therapy is associated with a delayed onset of CVD and prolonged survival, early and aggressive therapy should be initiated as soon as possible [6, 10]. The EAS has recommended LDL-C targets of $<100 \mathrm{mg} / \mathrm{dL}$ $(<2.5 \mathrm{mmol} / \mathrm{L})$ in children and $<70 \mathrm{mg} / \mathrm{dL}(<1.8 \mathrm{mmol} / \mathrm{L})$ in adults [10].

Statins, the first line of pharmacotherapy to lowering cholesterol level, effectively lower LDL$\mathrm{C} 10 \%$ to $25 \%$ in patients with $\mathrm{HoFH}[10,26]$, and even more (approximately $50 \%$ reduction of LDL-C) in those with HeFH [26]. The combination with ezetimibe (acholesterol absorption inhibitor) leads to additive $15-20 \%$ LDL-C reductions $[6,10]$. Other agents such as bile acid sequestrants, niacin, fibrates, and probucol can be considered. A clinical study of $\mathrm{HoFH}$ patients from South Africa found that statin use was associated with a $51 \%$ reduction in the risk of major CV events and a $66 \%$ reduction in the risk of death although the mean LDL-C levels in the patients were only reduced $26 \%$ [27].

Because of very high LDL-C levels in $\mathrm{HoFH}$, its target level is extremely difficult to achieve though cholesterol has been reduced [10]. The inability of standard lipid-lowering therapies to produce the necessary effect is further exacerbated by the fact that these agents work by increasing expression of LDLRs. Thus, lipoprotein apheresis should be considered in all patients with HoFH and should be initiated early. For example, the EAS guidelines recommend that apheresis should ideally be initiated by age 5 and not later than age 8 in children with $\mathrm{HoFH}$ [10]. Canadian guidelines recommend apheresis in adults with HoFH with LDL-C >329 $\mathrm{mg} / \mathrm{dL}(>8.5 \mathrm{mmol} / \mathrm{L}$ ) and in children (weighing $>15 \mathrm{~kg}$ or $>7$ years of age) with an LDL-C $>193$ $\mathrm{mg} / \mathrm{dL}$ ( $>5 \mathrm{mmol} / \mathrm{L}$ ) [6]. LDL apheresis selectively removes LDL-C without affecting immunoglobulins or other proteins with reductions of approximately $60 \%$ [18]. However, a rapid 
rebound in LDL-C is seen with levels returning to baseline within 2 to 4 weeks [18, 20]. Although there are no randomized trials evaluating the effect of apheresis on clinical outcomes, there is clinical evidence that apheresis can contribute to regression and/or stabilization of atherosclerotic plaque [10]. Limitations to the use of apheresis include lack of availability in some locations, high cost, long procedure duration, and the need to maintain vascular access [4]. It is recommended that patients on apheresis undergo routine monitoring to assess carotid atherosclerosis (carotid ultrasound), progression of aortic valve/root disease (echocardiography), and progression of coronary atherosclerosis (stress exercise test) [6].

\section{New pharmacologic therapies}

Recently, three novel agents have become available-mipomersen, lomitapide, and evolocumab-each with a unique mechanism of action. Two of these agents (mipomersen and lomitapide) target very low-density lipoprotein (VLDL) production, while the other (evolocumab) causes increased catabolism of LDL-C via LDLR recycling (Figure 2) [10].

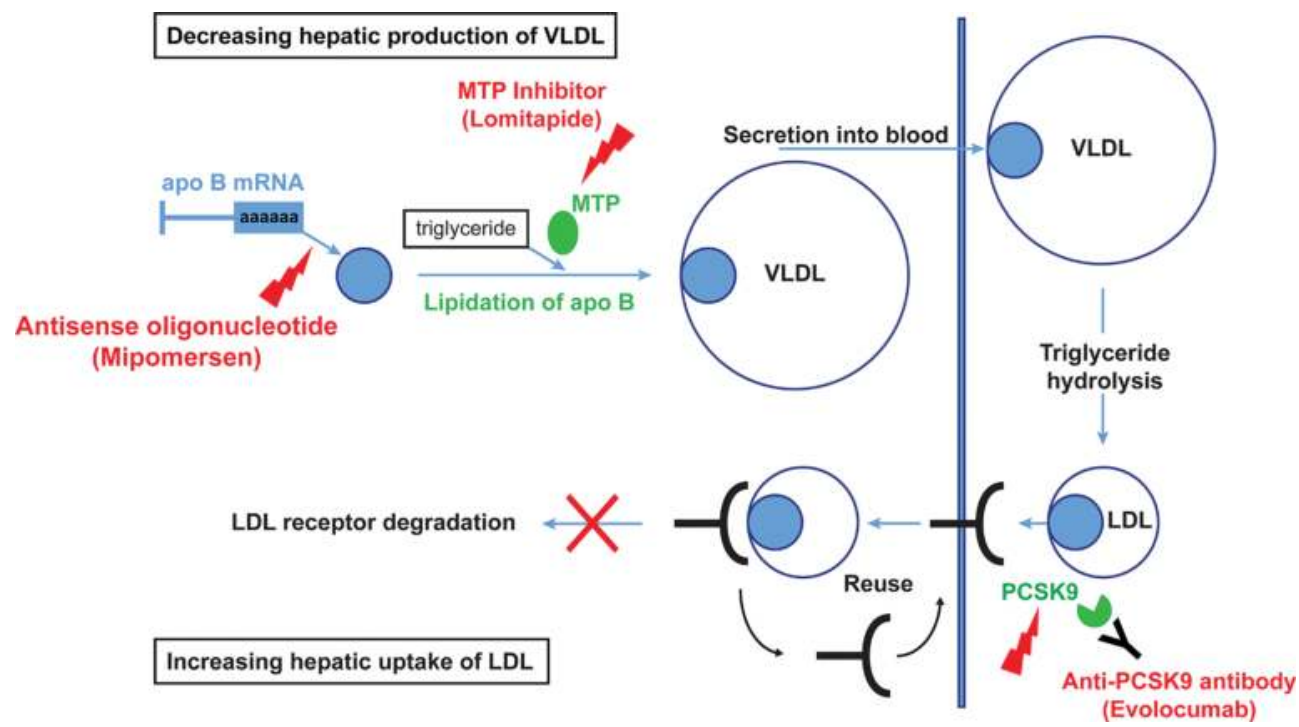

Figure 2. Mechanisms of action of mipomersen, lomitapide, and evolocumab. Modified from Cuchel et al. 2014 [10].

Properties of these agents are summarized in Table 1 [28-32] and are discussed in detail in the following sections. These agents produce additive LDL-C lowering when combined with other lipid-lowering therapies such as statins, ezetimibe, and apheresis [10] and represent promising approaches to the treatment of $\mathrm{HoFH}$ for those patients who cannot achieve LDL-C targets with conventional therapy. 


\begin{tabular}{|c|c|c|c|c|c|}
\hline Agent & MOA & Indication & Dosage and administration & $\begin{array}{l}\text { LDL-C } \\
\text { lowering }\end{array}$ & Adverse events \\
\hline Mipomersen $^{29}$ & $\begin{array}{l}\text { Oligonucleotide } \\
\text { inhibitor of } \\
\text { apolipoprotein B- } \\
100 \text { synthesis }\end{array}$ & $\begin{array}{l}\text { Adjunctive } \\
\text { therapy in } \\
\text { HoFH }\end{array}$ & $\begin{array}{l}\text { HoFH: } 200 \text { mg SC once } \\
\text { weekly }\end{array}$ & $25 \%$ & $\begin{array}{l}\text { Increased transaminases } \\
\text { Hepatic steatosis } \\
\text { Injection-site reactions }\end{array}$ \\
\hline $\begin{array}{l}\text { Lomitapide } \\
28,30,31\end{array}$ & $\begin{array}{l}\text { Microsomal } \\
\text { triglyceride transfer } \\
\text { protein inhibitor }\end{array}$ & $\begin{array}{l}\text { Adjunctive } \\
\text { therapy in } \\
\text { HoFH }\end{array}$ & $\begin{array}{l}\text { HoFH: Initiate at } 5 \mathrm{mg} / \mathrm{day} \text {, } \\
\text { titrating to max of } 60 \mathrm{mg} / \\
\text { day }\end{array}$ & $46 \%$ & $\begin{array}{l}\text { Increased transaminases } \\
\text { Hepatic steatosis }\end{array}$ \\
\hline Evolocumab $^{32}$ & PCSK9 inhibitor & $\begin{array}{l}\text { Adjunctive } \\
\text { therapy in } \\
\text { HeFH and } \\
\text { HoFH }\end{array}$ & $\begin{array}{l}\text { HeFH: } 140 \mathrm{mg} \text { SC every } 2 \\
\text { weeks or } 420 \mathrm{mg} \text { SC once } \\
\text { monthly } \\
\text { HoFH: } 420 \mathrm{mg} \text { SC once } \\
\text { monthly }\end{array}$ & $23 \%$ & $\begin{array}{l}\text { Nasopharyngitis, upper } \\
\text { respiratory tract } \\
\text { infection, influenza, back } \\
\text { pain, and injection-site } \\
\text { reactions }\end{array}$ \\
\hline
\end{tabular}

aBased on phase III trials in $\mathrm{HoFH}$;

${ }^{\mathrm{b}}$ Administered as three injections consecutively within 30 minutes.

$\mathrm{HeFH}$, heterozygous familial hypercholesterolemia; HoFH, homozygous familial hypercholesterolemia; LDL-C, lowdensity lipoprotein cholesterol; MOA, mechanisms of action; PCSK9, proprotein convertase subtilisin/kexin type 9; SC, subcutaneous.

Table 1. Novel agents for the treatment of HoFH.

\subsection{Mipomersen}

\subsubsection{Pharmacodynamics}

Apo $B$ is the primary protein of VLDL, intermediate density lipoprotein, and LDL and is essential for the production and catabolism of VLDL and LDL [33, 34]. Apo B is involved in the packaging and distribution of both dietary and endogenously produced cholesterol and triglycerides by lipoproteins [35]. The atherosclerotic potential of apo B is evidenced by the observation that apo B concentrations are highly predictive for atherosclerotic disease, including patients with $\mathrm{FH}[8,33]$.

Mipomersen is an antisense oligonucleotide against the mRNA of apo B-100, the primary ligand for the LDLR [33,34]. The drug reduces apo B mRNA translation, and thereby the synthesis of apo B by ribosomes, resulting in a reduction in the secretion of VLDL. Thus, mipomersen targets the production of LDL rather than its clearance (Figure 2) [34]. In animal 
models, species-specific inhibition of antisense apo B leads to reductions in apo B-100, LDL$\mathrm{C}$, and total cholesterol in a dose- and time-dependent manner [29, 35].

Mipomersen is readily absorbed after subcutaneous administration with the highest drug concentrations in the liver and kidney. Bioavailability ranges from $54 \%$ to $78 \%$ over a dose range of 50 to $400 \mathrm{mg}$ [29]. Elimination is primarily via metabolism by endonucleases and renal excretion (as parent drug and metabolites) and the half-life ranges from 1 to 2 months [29, 35]. In the United States, mipomersen is indicated as an adjunct to lipid-lowering medications and diet to reduce LDL-C, apo B, total cholesterol, and non-HDL-C in patients with HoFH [36]. The drug is administered once weekly by subcutaneous injection [29].

\subsubsection{Efficacy}

Based on its mechanism of action and its demonstrated activity in patients with hypercholesterolemia as either monotherapy or in combinations, it is reasonable that mipomersen would be effective in the treatment of $\mathrm{HoFH}$ [35]. In a phase II, open-label, study, mipomersen was administered in a dose-escalation fashion (50,100, 200, and $300 \mathrm{mg}$ ) to nine patients with HoFH. Patients received five doses over 2 weeks followed by weekly dosing through week 6 $(n=5)$ or week $13(n=4)$. At week 6, LDL-C reductions ranged from $0.5 \%$ to $36 \%$. By week 13 , the reductions ranged from $9.0 \%$ to $51.1 \%[29]$.

The phase III trial of mipomersen in patients with $\mathrm{HoFH}$ included 51 patients with clinical diagnosis or genetically confirmed HoFH [37]. Mean baseline LDL-C was $402 \mathrm{mg} / \mathrm{dL}$ (10.4 $\mathrm{mmol} / \mathrm{L})$. Patients who received maximally tolerated doses of lipid-lowering drug were randomized to receive mipomersen $200 \mathrm{mg}$ subcutaneously $(n=34)$ or placebo $(n=17)$ once weekly for 26 weeks [37]. The primary endpoint was the percent change in LDL-C concentration from baseline. Secondary end points were changes from baseline in apo B, total cholesterol, and non-HDL-C concentrations. At 26 weeks, mipomersen-treated patients achieved significant reductions in all primary and secondary endpoints versus placebo: LDL-C ( $-24.7 \%)$, apo B $(-26.8 \%)$, total cholesterol (-21.2\%), and non-HDLC (-24.5\%). By comparison, reductions for those in the placebo group were: LDL-C $(-3.3 \%)$, apo B $(-2.5 \%)$, total cholesterol $(-2.0 \%)$, and non-HDL-C $(-2.9 \%)$. In addition, mipomersen was also associated with substantial reductions in $\operatorname{Lp}(\mathrm{a})(-31.1 \%)$, triglycerides $(-17.4 \%)$, and VLDL $(-17.4 \%)$, and a significant increase in HDL-C (+15.1\%). Notably, there was substantial variability in the reduction of LDL-C concentrations among $\mathrm{HoFH}$ patients receiving mipomersen with values ranging from $+2 \%$ to $-82 \%$. The magnitude of treatment effect was independent of baseline LDL-C, age, race, or sex in multivariate analysis [37].

\subsubsection{Safety/tolerability}

In the phase III HoFH trial, the most common adverse events among patients with HoFH were injection-site reactions $(76 \%)$, flu-like symptoms $(29 \%)$, nausea $(18 \%)$, headache $(15 \%)$, and chest pain (12\%). Injection-site reactions included erythema (56\%), hematoma (35\%), pain $(35 \%)$, pruritus $(29 \%)$, discoloration $(29 \%)$, macule $(15 \%)$, papule $(12 \%)$, and swelling $(12 \%)$. Similar rates of injection-site reactions were observed in pooled data from other clinical trials 
with rates of $84 \%$ and $33 \%$, respectively, for those in the mipomersen and placebo groups [29]. Most reactions were of mild to moderate severity with only $5 \%$ discontinuing treatment because of an injection-site reaction. In pooled phase III trials that included all patients with hypercholesterolemia, 30\% of patients experienced flu-like symptoms (e.g., pyrexia, chills, myalgia, arthralgia, malaise, fatigue) compared with $16 \%$ of those receiving placebo [29].

Laboratory abnormalities in the phase III HoFH trial were primarily characterized by elevated liver transaminases. Alanine aminotransferase (ALT) increases of $\geq 1$ but $\leq 3$ times the upper limit of normal (ULN) were observed in $50 \%$ of patients in the mipomersen groups but was similar to that seen with placebo (53\%). However, increased ALT of $\geq 3 \times$ ULN was seen in $12 \%$ of mipomersen-treated patients but none of the placebo-treated patients [37]. In the pooled phase III trials, $8.4 \%$ of patients receiving mipomersen experienced an elevated ALT $>3 \times$ ULN on two consecutive occasions at least 7 days apart compared to $0.0 \%$ of placebo-treated patients [29]. These ALT changes were generally associated with lesser elevations of aspartate aminotransferase (AST). Mipomersen was also associated with an increase in hepatic fat in $9.6 \%$ of patients compared with $0.02 \%$ of placebo-treated patients. However, this increase was not accompanied by changes in patient weight, plasma glucose, or HbA1c, suggesting that there is no associated increased risk of metabolic syndrome. It is suggested that the hepatic steatosis and elevated transaminase concentrations are inherent consequences of attenuating apo B production. Nevertheless, mipomersen carries a black box warning for the risk of hepatotoxicity (i.e., increased transaminases and hepatic steatosis) and the drug is only available in the United States via a Risk Evaluation and Mitigation Strategy program [29].

\subsection{Lomitapide}

\subsubsection{Pharmacodynamics}

The microsomal triglyceride transfer protein (MTP) is an intracellular lipid-transfer protein located in the lumen of the endoplasmic reticulum. It is responsible for binding and moving individual lipid molecules between membranes. MTP is a major mediator of the assembly and secretion of apo B-containing lipoproteins such as VLDL from the liver, which is converted into LDL-C, and chylomicrons, which contain dietary cholesterol and triglycerides, from the intestine $[30,31,38]$. The rare genetic condition abetalipoproteinemia provides insight into the importance of MTP in lipid handling and transport. Abetalipoproteinemia is characterized by loss-of-function mutations in the gene encoding MTP (i.e., MTTP) and is associated with marked hypocholesterolemia and an absence of apo B-containing lipoproteins in the plasma [35]. Lack of functional MTP in abetalipoproteinemia results in the inability to load apo B with lipoproteins and the targeted proteasomal degradation of apo B. This leads to a loss of intestinal secretion of chylomicrons and liver secretion of VLDL and a consequent lack of LDL-C in the plasma [35]. Thus, inhibition of MTP is a potentially powerful therapeutic target to reduce the production of apo B-containing lipoproteins, particularly VLDL (the precursor of LDL-C) [30].

Lomitapide is a small molecule that inhibits MTP action. By binding directly to MTP, lomitapide inhibits the synthesis of triglyceride-rich chylomicrons in the intestine and VLDL in the 
liver, with a resulting reduction in plasma LDL-C [39]. The mechanism of action of lomitapide in inhibiting MTP is illustrated in Figure 2.

Oral absorption of lomitapide is poor with an absolute bioavailability of $7 \%$, thought to be due to a first-pass effect. Lomitapide pharmacokinetics is approximately dose proportional after single oral doses of 10-100 mg. The drug is extensively metabolized in the liver and has a terminal half-life of 39.7 hours [28, 30]. Lomitapide is indicated in the United States and the European Union as an adjunct to a low-fat diet and other lipid-lowering treatments, including LDL apheresis where available, to reduce LDL-C, total cholesterol, apo B, and non-HDL-C in patients with $\mathrm{HoFH}[28,39]$.

\subsubsection{Efficacy}

An initial study in 18 patients with HoFH evaluated the addition of lomitapide to usual lipidlowering therapy, including apheresis [40]. The dose of lomitapide was gradually titrated during the first 14-18 weeks to a target dose of $60 \mathrm{mg} /$ day $(80 \mathrm{mg} /$ day if LDL and safety criteria were met). The mean overall LDL-C reduction was $44 \%$ at 6 months compared with baseline but the individual values ranged from an increase in LDL-C of $19 \%$ to a reduction of $93 \%$, indicating a wide variability of effect. Four patients achieved an LDL-C $<100 \mathrm{mg} / \mathrm{dL}(<2.6$ $\mathrm{mmol} / \mathrm{L})$ and another two achieved levels $<170 \mathrm{mg} / \mathrm{dL}(<4.4 \mathrm{mmol} / \mathrm{L})$ [40].

The pivotal phase III open-label trial included 29 patients with HoFH based on clinical criteria or documented genetic mutations [41]. Upon enrollment, patients were required to enter a 6week run-in phase in which patients were initiated on concomitant lipid-lowering therapy (including apheresis), vitamin E, essential fatty acids, and a low-fat diet. Patients then entered a 26-week efficacy phase where lomitapide was initiated at $5 \mathrm{mg} /$ day and titrated (at 4-week intervals) up to a maximum of $60 \mathrm{mg} /$ day. Following the efficacy phase, patients continued lomitapide therapy in a 52-week safety phase. Mean baseline total cholesterol and LDL-C levels were $429 \mathrm{mg} / \mathrm{dL}(11.1 \mathrm{mmol} / \mathrm{L})$ and $336 \mathrm{mg} / \mathrm{dL}(8.7 \mathrm{mmol} / \mathrm{L})$, respectively [41]. Twenty-three of 29 patients completed both the efficacy phase ( 26 weeks) and safety phase (52 weeks). At the end of 26 weeks, patients achieved statistically significant mean reductions from baseline in total cholesterol $(-46 \% ; P<0.0001)$ and LDL-C $(-50 \% ; P<0.0001)$ [41]. The large majority of patients $(n=19 / 23[83 \%])$ achieved LDL-C reductions $>25 \%$ and one-half $(n=12 / 23)$ had a $>50 \%$ reduction [41]. Furthermore, 8 patients achieved LDL-C concentrations $<100 \mathrm{mg} / \mathrm{dL}(<2.6$ $\mathrm{mmol} / \mathrm{L}$ ). Based on these LDL-C reductions, three patients permanently discontinued apheresis and three permanently increased the time interval between apheresis treatments. Significant reductions from baseline were also seen for VLDL cholesterol ( $-45 \%)$, non-HDL-C $(-50 \%)$, triglycerides $(-45 \%)$, and apo B $(-49 \%)$. Lipid lowering was independent of the use of apheresis, suggesting that apheresis does not affect the lipid-lowering efficacy of lomitapide [42]. These reductions were maintained throughout the 52-week safety phase with reductions of $35 \%$ and $38 \%$, respectively, for total cholesterol and LDL-C despite changes in concomitant lipid-lowering therapy [41]. Nineteen of the 23 patients who competed the efficacy and safety phases entered a long-term extension study [43,44]. As of 2015, the median duration of treatment was 5.1 years [43]. At 126 weeks, mean LDL-C levels were reduced by $46 \%$. Similar 
reductions were also observed in apo B (-54\%), non-HDL-C (-47\%), VLDL cholesterol $(-37 \%)$, and triglycerides $(-38 \%)[43,44]$.

Additional evidence of the efficacy of lomitapide in HoFH comes from a Japanese trial [45] and the Lomitapide Observational Worldwide Evaluation Registry (LOWER) [45, 46]. The Japanese trial included nine patients with a mean baseline LDL-C of $199 \mathrm{mg} / \mathrm{dL}(5.2 \mathrm{mmol} / \mathrm{L})$, which was reduced to $118 \mathrm{mg} / \mathrm{dL}(3.1 \mathrm{mmol} / \mathrm{L})$ at week $26(-42 \%)$ [45]. Significant reductions were also seen for total cholesterol $(-32 \%)$, non-HDL-C $(-40 \%)$, VLDL $(-42 \%)$, apo B $(-45 \%)$, and triglycerides $(-42 \%)$ [45]. LOWER is a noninterventional registry open to lomitapidetreated patients that is designed to evaluate the long-term safety and efficacy of lomitapide in clinical practice and is eventually expected to enroll at least 300 patients and follow them for at least 10 years [47]. As of March 2015, 84 patients had enrolled in LOWER, with all but one from the United States [46]. Titration of lomitapide occurred slower than in the pivotal phase III trial, with a mean dose of $10 \mathrm{mg}$ reached only after 12 months. The mean reduction in LDLC at month 4 was $42 \%$, with $38 \%$ of patients achieving a reduction of at least $50 \%$ at 6 months $[46,47]$.

\subsubsection{Safety/tolerability}

Oral lomitapide was generally well tolerated in patients with HoFH. Although the majority of patients experienced an adverse event in the phase III trial ( $n=27 / 29$ [93\%] in the efficacy phase; $n=21 / 23$ [91\%] in the safety phase), most events were mild to moderate in intensity [41]. The most common adverse events were gastrointestinal in nature, with 27/29 patients in the efficacy phase and 21/23 patients in the safety phase experiencing a gastrointestinal event [41]. The most common events in the phase III trial were gastrointestinal in nature (27 patients during the efficacy phase and 17 during the safety phase), most commonly manifested as diarrhea, nausea, dyspepsia, and vomiting [41,43]. Three patients discontinued treatment due to a gastrointestinal event [41]. The incidence of gastrointestinal events decreased during the extension phase: diarrhea (42\%), nausea (32\%), vomiting (26\%), and dyspepsia (11\%) [43].

Ten patients in the phase III trial had elevated levels of ALT, AST, or both $>3 \times$ ULN at least once during the trial, and four patients had elevations at least $5 \times \mathrm{ULN}$ [41]. No patient discontinued treatment permanently because of these elevations and all were managed by either dose reduction or temporary interruption of lomitapide [41, 43]. In the LOWER registry, elevated transaminase levels $\geq 3 \times$ ULN were observed in only 16 patients (19\%) [46].

Among the 20 patients from the phase III trials with evaluable nuclear magnetic resonance spectroscopy data, hepatic fat increased from $1 \%$ at baseline to $8.6 \%$ at the end of week 26 and $8.3 \%$ at week 78 [41]. Hepatic fat continued to increase through the extension trial [43], although the accumulation of fat appears to be reversible after discontinuation of lomitapide [39]. Whether this fat accumulation is a risk factor for the development of steatohepatitis and cirrhosis is currently unknown. No cases of cirrhosis or late-stage liver disease have been identified in the long-term extension studies [43]. 


\subsection{Evolocumab}

\subsubsection{Pharmacodynamics}

PCSK9 is a key regulator of LDLR function. When PCSK9 binds to the LDLR, LDLR degradation is enhanced in the liver, thereby increasing LDL-C plasma concentrations [4].Although some patients with HoFH have no LDLR function, up to $75 \%$ have residual activity (between $2 \%$ and 25\%) [2]. Patients with HoFH also have increased PCSK9 function. Among patients with residual LDLR function, PCSK9 inhibition may be useful for lowering LDL-C [2]. Evolocumab is a human immunoglobulin G2 monoclonal antibody directed against human PCSK9. By binding to PCSK9, evolocumab inhibits circulating PCSK9 from binding to the LDLR, preventing PCSK9-mediated LDLR degradation and permitting LDLR to recycle back to the liver cell surface. This increases the number of LDLRs available to clear LDL from the blood, thereby lowering LDL-C level (Figure 2) [32, 48, 49].

\subsubsection{Efficacy}

The addition of evolocumab to stable lipid-lowering therapy was evaluated in an open-label pilot trial in eight patients with LDLR-negative or LDLR-defective HoFH [32]. Patients received subcutaneous evolocumab 420 mg every 4 weeks for 12 weeks, maintained for an additional 12 weeks at 4 -week intervals, and then $420 \mathrm{mg}$ of evolocumab every 2 weeks for an additional 12 weeks [32]. All eight patients had LDLR mutations, with six patients having defective receptor status (i.e., residual LDLR function) and two having negative LDLR function. Mean baseline LDL-C was $441 \mathrm{mg} / \mathrm{dL}$ (11.4 mmol/L) [32]. After 12 weeks of every 4-week dosing, mean LDL-C decreased by a mean of $17 \%$ (range, $+5 \%$ to $-44 \%$ ). The two patients with negative LDLR activity did not achieve reductions in LDL-C [32]. After 12 weeks of every 2-week dosing, mean LDL-C was reduced by $14 \%$, again with no reductions in the two patients that were LDLR-negative. Apo B was reduced by $14.9 \%$ and $12.5 \%$ by the 4 -week and 2-week dosing schedules and $\mathrm{Lp}(\mathrm{a})$ was reduced by $11.7 \%$ and $18.6 \%$, respectively, by the two schedules. However, there was little change in triglycerides, HDL-C, or apolipoprotein A1 with either schedule [32].

The pivotal randomized, phase III, double-blind, placebo-controlled trial included 49 patients with HoFH on stable lipid-lowering therapy (but not apheresis) for at least 4 weeks. Patients were randomized in a 2:1 ratio to receive evolocumab $420 \mathrm{mg}$ or placebo every 4 weeks [48]. LDLR mutations in both alleles were present in 45 of 48 patients (94\%), with 22 of these having the same mutation in both alleles (true $\mathrm{HoFH}$ ) and 23 having different mutations in each LDLR allele (i.e., compound heterozygous $\mathrm{FH}$ ) [48]. One patient receiving evolocumab had LDLR receptor-negative mutations in both alleles and another had autosomal recessive hypercholesterolemia. The mean decrease in ultracentrifugation LDL-C was $23.1 \%$ for those receiving evolocumab compared with a 7.9\% increase for the placebo group (primary endpoint) [48]. Evolocumab was also associated with a $19.2 \%$ reduction in apo B at week 12 , although changes in Lp(a), HDL-C, and triglycerides were not significantly different relative to placebo [48]. Response to evolocumab correlated with the underlying genetic cause of $\mathrm{HoFH}$, with a greater reduction in LDL-C among those with two LDLR-defective mutations than in those with even 
a single LDLR-negative mutation. However, among the 20 patients receiving evolocumab who had defects in either one or both alleles, a $29.5 \%$ reduction in ultracentrifugation LDL-C was achieved [48]. The patient with LDLR-negative mutations in both alleles and the one with autosomal recessive hypercholesterolemia did not respond to evolocumab (LDL-C levels increased by $3-10 \%$ ) [48].

The efficacy of evolocumab in combination with apheresis is under evaluation in the Trial Assessing Long Term Use of PCSK9 Inhibition in Subjects with Genetic LDL Disorders (TAUSSIG) in patients with severe FH not controlled with current lipid therapy [50]. Patients received evolocumab $420 \mathrm{mg}$ and apheresis every 2 weeks. An interim analysis found that evolocumab was associated with a mean reduction of $17 \%$ in LDL-C at week $12(n=24)$ and $20 \%$ at week $24(n=12)$ [50]. Four patients were able to stop or decrease the frequency of apheresis. The three patients with LDLR-negative mutations in both alleles did not respond to evolocumab. Evolocumab is indicated in the United States and EU as an adjunct to diet and other LDL-lowering therapies for the treatment of patients with $\mathrm{HoFH}$ who require additional lowering of LDL-C.

\subsubsection{Safety/tolerability}

In the phase III trial in patients with $\mathrm{HoFH}$, the most common adverse events among those receiving evolocumab were upper respiratory tract infection $(9 \%)$, influenza $(9 \%)$, gastroenteritis $(6 \%)$, nasopharyngitis $(6 \%)$, and increased ALT or AST $\geq 3 \times$ ULN [48]. There were no adverse event-related treatment discontinuations. These rates of adverse events are generally consistent with those seen in other large randomized trials evaluating evolocumab in the treatment of hypercholesterolemia [49]. Immunogenicity appears to be uncommon, with only $0.1 \%$ of patients in pooled clinical trials testing positive for binding antibody development. There was no evidence of neutralizing antibodies and no evidence that the presence of antidrug antibodies impacted the pharmacokinetic profile, clinical response, or safety of evolocumab [49].

\section{Conclusions}

$\mathrm{HoFH}$ is a rare disease that is underdiagnosed and undertreated and is associated with substantial morbidity and mortality. Early diagnosis and aggressive therapy are the cornerstones of the management of HoFH. Until recently, therapeutic options were limited and insufficient to get patients to their treatment goals. The availability of novel pharmacologic agents provides clinicians with additional treatment options in this difficult-to-treat population. Figure 3 summarizes the suggested treatment algorithm of the EAS for patients with HoFH [10].

This algorithm highlights the novel treatment options that will allow greater reductions in lipid levels in $\mathrm{HoFH}$ patients and let them achieve their target goals. It is hoped and expected 
that these expanded options will ultimately translate into improvements in clinical outcomes including a decrease in CV events and CVD-related mortality.

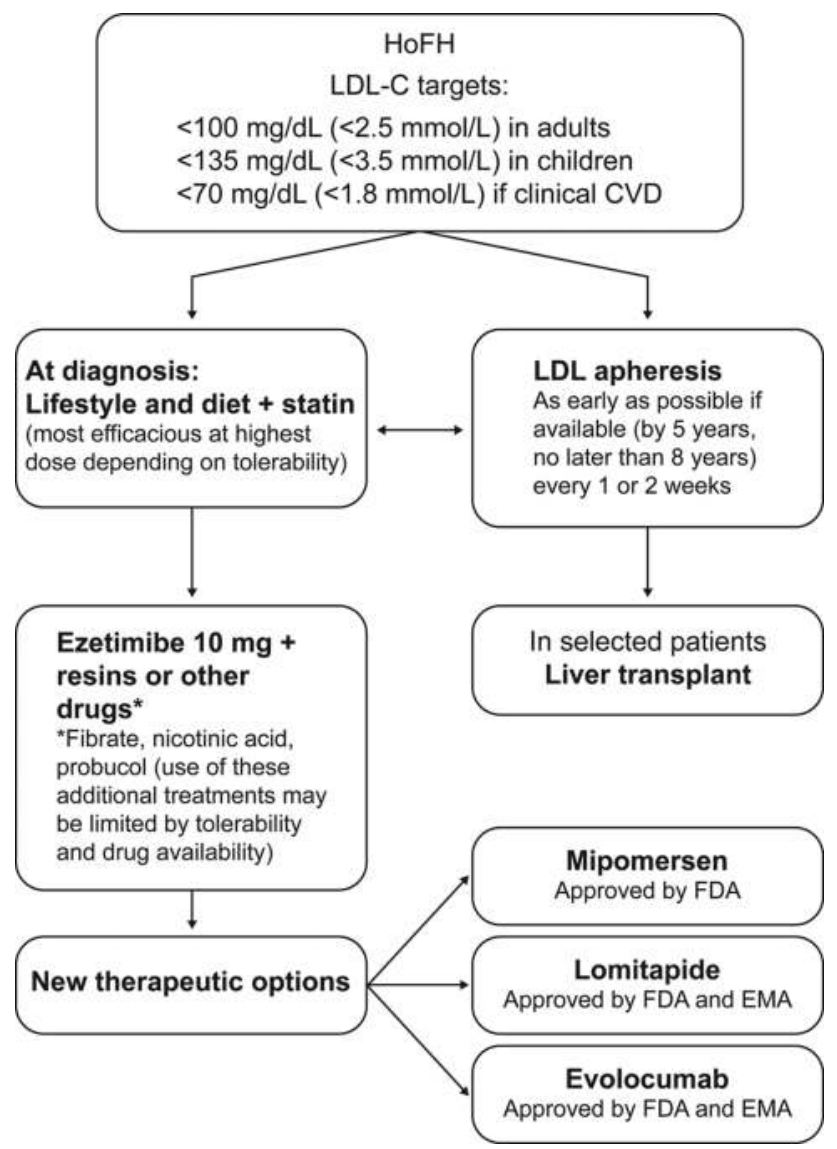

Figure 3. European Atherosclerosis Society treatment algorithm for the management of HoFH. Modified from Cuchel et al. 2014 [10].

\section{Author details}

Min-Ji Charng

Address all correspondence to: mjcharng@vghtpe.gov.tw

Division of Cardiology, Taipei Veterans General Hospital and National Yang-Ming University, Taipei, Taiwan, R.O.C. 


\section{References}

[1] Sniderman AD, Tsimikas S, Fazio S. The severe hypercholesterolemia phenotype: clinical diagnosis, management, and emerging therapies. J Am Coll Cardiol. 2014;63:1935-1947.

[2] Chiou KR, Charng MJ. Genetic diagnosis of familial hypercholesterolemia in Han J Clin Lipidol. 2016;10:490-496.

[3] Marais AD, Blom DJ. Recent advances in the treatment of homozygous familial hypercholesterolaemia. Curr Opin Lipidol. 2013;24:288-294.

[4] Farnier M, Bruckert E. Severe familial hypercholesterolaemia: current and future management. Arch Cardiovasc Dis. 2012;105:656-665.

[5] Singh S, Bittner V. Familial hypercholesterolemia-epidemiology, diagnosis, and screening. Curr Atheroscler Rep. 2015;17:482-485.

[6] Genest J, Hegele RA, Bergeron J, Brophy J, Carpentier A, Couture P, et al. Canadian Cardiovascular Society position statement on familial hypercholesterolemia. Can J Cardiol. 2014;30:1471-1481.

[7] Nordestgaard BG, Chapman MJ, Humphries SE, Ginsberg HN, Masana L, Descamps OS, et al. Familial hypercholesterolaemia is underdiagnosed and undertreated in the general population: guidance for clinicians to prevent coronary heart disease: consensus statement of the European Atherosclerosis Society. Eur Heart J. 2013;34:3478-3490.

[8] Raal FJ, Santos RD. Homozygous familial hypercholesterolemia: current perspectives on diagnosis and treatment. Atherosclerosis. 2012;223:262-268.

[9] Horton JD, Cohen JC, Hobbs HH. PCSK9: a convertase that coordinates LDL catabolism. J Lipid Res. 2009;50(Suppl):S172-S177.

[10] Cuchel M, Bruckert E, Ginsberg HN, Raal FJ, Santos RD, Hegele RA, et al. Homozygous familial hypercholesterolaemia: new insights and guidance for clinicians to improve detection and clinical management. A position paper from the Consensus Panel on Familial Hypercholesterolaemia of the European Atherosclerosis Society. Eur Heart J. 2014;35:2146-2157.

[11] Fahed AC, Safa RM, Haddad FF, Bitar FF, Andary RR, Arabi MT, et al. Homozygous familial hypercholesterolemia in Lebanon: a genotype/phenotype correlation. Mol Genet Metab. 2011;102:181-188.

[12] Kusters DM, Huijgen R, Defesche JC, Vissers MN, Kindt I, Hutten BA, et al. Founder mutations in the Netherlands: geographical distribution of the most prevalent mutations in the low-density lipoprotein receptor and apolipoprotein B genes. Neth Heart J. 2011;19:175-182. 
[13] Mabuchi H, Nohara A, Noguchi T, Kobayashi J, Kawashiri MA, Tada H, et al. Molecular genetic epidemiology of homozygous familial hypercholesterolemia in the Hokuriku district of Japan. Atherosclerosis. 2011;214:404-407.

[14] Moorjani S, Roy M, Gagne C, Davignon J, Brun D, Toussaint M, et al. Homozygous familial hypercholesterolemia among French Canadians in Quebec Province. Arteriosclerosis. 1989;9:211-216.

[15] Seftel HC, Baker SG, Sandler MP, Forman MB, Joffe BI, Mendelsohn D, et al. A host of hypercholesterolaemic homozygotes in South Africa. Br Med J. 1980;281:633-636.

[16] Feldman DI, Blaha MJ, Santos RD, Jones SR, Blumenthal RS, Toth PP, et al. Recommendations for the management of patients with familial hypercholesterolemia. Curr Atheroscler Rep. 2015;17:473. DOI: 10.1007/s11883-014-0473-6

[17] Alonso R, Mata P, Zambon D, Mata N, Fuentes-Jimenez F. Early diagnosis and treatment of familial hypercholesterolemia: improving patient outcomes. Expert Rev Cardiovasc Ther. 2013;11:327-342. DOI: 10.1586/erc.13.7

[18] Mombelli G, Pavanello C. Novel therapeutic strategies for the homozygous familial hypercholesterolemia. Recent Pat Cardiovasc Drug Discov. 2013;8:143-150. DOI: $10.2174 / 15748901112079990001$

[19] Minhas R, Humphries SE, Qureshi N, Neil HA. Controversies in familial hypercholesterolaemia: recommendations of the NICE Guideline Development Group for the identification and management of familial hypercholesterolaemia. Heart. 2009;95:584587. DOI: $10.1136 /$ hrt.2008.162909

[20] Robinson JG. Management of familial hypercholesterolemia: a review of the recommendations from the National Lipid Association Expert Panel on Familial Hypercholesterolemia. J Manag Care Pharm. 2013;19:139-149.

[21] Gouni-Berthold I, Berthold HK. Familial hypercholesterolemia: etiology, diagnosis and new treatment options. Curr Pharm Des. 2014;20:6220-6229.

[22] Reiner Z, Catapano AL, De Backer G, Graham I, Taskinen MR, Wiklund O, et al. ESC/EAS Guidelines for the management of dyslipidaemias: the Task Force for the management of dyslipidaemias of the European Society of Cardiology (ESC) and the European Atherosclerosis Society (EAS). Eur Heart J. 2011;32:1769-1818. DOI: 10.1093/ eurheartj/ehr158

[23] Ray KK, Kastelein JJ, Boekholdt SM, Nicholls SJ, Khaw KT, Ballantyne CM, et al. The ACC/AHA 2013 guideline on the treatment of blood cholesterol to reduce atherosclerotic cardiovascular disease risk in adults: the good the bad and the uncertain: a comparison with ESC/EAS guidelines for the management of dyslipidaemias 2011. Eur Heart J. 2014;35:960-968. DOI: 10.1093/eurheartj/ehu107

[24] Stone NJ, Robinson JG, Lichtenstein AH, Bairey Merz CN, Blum CB, Eckel RH, et al. 2013 ACC/AHA guideline on the treatment of blood cholesterol to reduce atheroscler- 
otic cardiovascular risk in adults: a report of the American College of Cardiology/ American Heart Association Task Force on Practice Guidelines. Circulation. 2014;129:S1-S45. DOI: 10.1161/01.cir.0000437738.63853.7a

[25] National Institute on Clinical Care and Excellence (NICE). Familial hypercholesterolaemia: identifification and management.2008. Available from: http://www.nice.org.uk/ guidance/cg71. [Accessed Nov 23, 2015]

[26] Bruckert E. Recommendations for the management of patients with homozygous familial hypercholesterolaemia: overview of a new European Atherosclerosis Society consensus statement. Atheroscler Suppl. 2014;15:26-32.

[27] Raal FJ, Pilcher GJ, Panz VR, van Deventer HE, Brice BC, Blom DJ, et al. Reduction in mortality in subjects with homozygous familial hypercholesterolemia associated with advances in lipid-lowering therapy. Circulation. 2011;124:2202-2207.

[28] JUXTAPID (lomitapide): package insert. Cambridge, MA: Aegerion Pharmaceuticals, Inc.; 2015.

[29] McGowan MP, Moriarty PM, Backes JM. The effects of mipomersen, a second-generation antisense oligonucleotide, on atherogenic (apoB-containing) lipoproteins in the treatment of homozygous familial hypercholesterolemia. Clin Lipidol. 2014;9:487-503.

[30] Perry CM. Lomitapide: a review of its use in adults with homozygous familial hypercholesterolemia. Am J Cardiovasc Drugs. 2013;13:285-296.

[31] Raal FJ. Lomitapide for homozygous familial hypercholesterolaemia. Lancet. 2013;381:7-8.

[32] Stein EA, Honarpour N, Wasserman SM, Xu F, Scott R, Raal FJ. Effect of the proprotein convertase subtilisin/kexin 9 monoclonal antibody, AMG 145, in homozygous familial hypercholesterolemia. Circulation. 2013;128:2113-2120.

[33] Parhofer KG. Mipomersen: evidence-based review of its potential in the treatment of homozygous and severe heterozygous familial hypercholesterolemia. Core Evid. 2012;7:29-38.

[34] Patel N, Hegele RA. Mipomersen as a potential adjunctive therapy for hypercholesterolemia. Expert Opin Pharmacother. 2010;11:2569-2572.

[35] Rader DJ, Kastelein JJ. Lomitapide and mipomersen: two first-in-class drugs for reducing low-density lipoprotein cholesterol in patients with homozygous familial hypercholesterolemia. Circulation. 2014;129:1022-1032.

[36] KYNAMRO (mipomersen sodium): package insert. Cambridge, MA: Genzyme Corporation; 2015.

[37] Raal FJ, Santos RD, Blom DJ, Marais AD, Charng MJ, Cromwell WC, et al. Mipomersen, an apolipoprotein B synthesis inhibitor, for lowering of LDL cholesterol concentrations 
in patients with homozygous familial hypercholesterolaemia: a randomised, doubleblind, placebo-controlled trial. Lancet. 2010;375:998-1006.

[38] Liao W, Hui TY, Young SG, Davis RA. Blocking microsomal triglyceride transfer protein interferes with apoB secretion without causing retention or stress in the ER. J Lipid Res. 2003;44:978-985.

[39] Lyseng-Willliamson KA, Perry CM. Lomitapide: a guide to its use in adults with homozygous familial hypercholesterolaemia in the EU. Drugs Ther Perspect. 2013;29:373-378.

[40] Cuchel M, Meagher E, Marais AD, Blom DJ, Theron HD, Baer AL, et al. Abstract 1077: a phase III study of microsomal triglyceride transfer protein inhibitor lomitapide (AEGR-733) in patients with homozygous familial hypercholesterolemia: interim results at 6 months [Abstract]. Circulation. 2009;120:S441.

[41] Cuchel M, Meagher EA, du Toit Theron H, Blom DJ, Marais AD, Hegele RA, et al. Efficacy and safety of a microsomal triglyceride transfer protein inhibitor in patients with homozygous familial hypercholesterolaemia: a single-arm, open-label, phase 3 study. Lancet. 2013;381:40-46.

[42] Averna M, Cuchel M, Meagher E, Du H, Theron T, Blom DJ, et al. Apheresis treatment does not affect the lipid-lowering efficacy of lomitapide, a microsomal triglyceride transfer protein inhibitor, in patients with homozygous familial hypercholesterolemia (HoFH). In: Deutschen Atherosklerosekongress 2012; 7-8 December 2012; Munich, Germany. Perfusion. 2012.

[43] Blom DJ, Averna M, Meagher E, Theron H, Sirtori C, Hegele RA, et al. Long-term efficacy and safety of lomitapide for the treatment of homozygous familial hypercholesterolemia: results of the phase 3 extension trial. In: American Heart Association; $7-$ 11 November 2015; Orlando, FL.

[44] Cuchel M, Blom DJ, Averna MR, Meagher EA, Theron HD, Sirtori CR, et al. Sustained LDL-C lowering and stable hepatic fat levels in patients with homozygous familial hypercholesterolemia treated with the microsomal triglyceride transfer protein inhibitor, lomitapide: results of an ongoing long-term extension study [Abstract]. Circulation. 2013;128:A16516.

[45] Harada-Shiba M, Ikewaki K, Nohara A, Yanagi K, Otsubo Y, Foulds P, et al. Efficacy and safety of lomitapide in Japanese patients with homozygous familial hypercholesterolemia on cucurrent lipid-lowering therapy [Abstract]. Circulation. 2015;132:A12468.

[46] Blom DJ, Kastelein JJ, Larrey D, Makris L, Schwamiein C, Phillips H, et al. Lomitapide Observational Worldwide Evaluation Registry (LOWER): one-year data [Abstract]. Circulation. 2015;132:A10818. 
[47] Blom DJ, Fayad ZA, Kastelein JJP, Larrey D, Makris L, Schwamiein C, et al. LOWER, a registry of lomitapide-treated patients with homozygous familial hypercholesterolemia: rationale and design. J Clin Lipidol. 2015;10:273-282.

[48] Raal FJ, Honarpour N, Blom DJ, Hovingh GK, Xu F, Scott R, et al. Inhibition of PCSK9 with evolocumab in homozygous familial hypercholesterolaemia (TESLA Part B): a randomised, double-blind, placebo-controlled trial. Lancet. 2015;385:341-350.

[49] REPATHA (evolocumab): package insert. Thousand Oaks, CA: Amgen, Inc.; 2015.

[50] Bruckert E, Blaha V, Stein EA, Raal FJ, Kurtz CE, Honarpour N, et al. Trial assessing long-term use of PCSK9 inhibition in patients with genetic LDL disorders (TAUSSIG): efficacy and safety in patients with homozygous familial hypercholesterolemia receiving lipid apheresis [Abstract]. Circulation. 2014;130:A17016. 
